\title{
A smarandache completely prime ideal with respect to an element of near ring
}

\author{
Kareem Abass Layith AL-Ghurabi \\ Department of Mathematics, College of Education for Pure Sciences, University of Babylon \\ E-mail: kareemalghurabi@yahoo.com
}

Copyright $\odot 2014$ Kareem Abass Layith AL-Ghurabi. This is an open access article distributed under the Creative Commons Attribution License, which permits unrestricted use, distribution, and reproduction in any medium, provided the original work is properly cited.

\begin{abstract}
In this paper we introduce the notion of a smarandache completely prime ideal with respect to an element belated to a near field of a near ring N (b-s-c.p.i) of N. We study some properties of this new concept and link it with some there types of ideals of a near ring.

Keywords: Smarandache Completely Prime, Near Ring.

\section{Introduction}

In 1905, L.E. Drckson began the study of a near ring and later in 1930; Wieland has investigated it [1]. In 1977, G.Pilz, introduced the notion of a prime ideal of near ring [1]. In 1988, N.G. Groenewald introduced of a completely prime ideal of a near ring [5]. In 2002, W.B. Vasanth Kandasamy study samaradache near ring, (samaradache ideal, of a near ring [7]. In 2012 H.H. Abbass and M.A.Mohommed introduced the notion of a completely prime ideal with respect to an element of a near ring [3].

In this work, we introduce a Samaradache completely prime ideal with respect to an element related to a near field of near ring as we mentioned in the abstract.
\end{abstract}

\section{Preliminaries}

In this section, we review some basic concepts about a near ring, and some types of fields of a near rind that We need in our work.

Definition 2.1 [1]: A left near ring is a set $N$ together with two binary operations "+ " and". " such that

1. $(\mathrm{N},+)$ Is a group (not necessarily abelian),

2. $(\mathrm{N},$.$) Is a semi group?$

3. $\mathrm{n}_{1} \cdot\left(\mathrm{n}_{2}+\mathrm{n}_{3}\right)=\mathrm{n}_{1} \mathrm{n}_{2}+\mathrm{n}_{1} \mathrm{n}_{3}$, for all $\mathrm{n}_{1}, \mathrm{n}_{2}, \mathrm{n}_{3}, \in \mathrm{N}$.

Definition 2.2 [2]: The left near ring is called a zero symmetric if $0 . x=0$, for allx $\in N$.

Definition 2.3[7]: Left $(N,+,$.$) be a near-ring. A normal subgroup I of (N,+)$ is called a left ideal of $N$ if

1. $\quad$ N. I $\subseteq$ I

2. for all $n, n_{1} \in N$ and for all $i \in I$,

$(n+i) \cdot n-n_{1} \cdot n \in I$

Remark 2.4: If $\mathrm{N}$ is a left near ring, then $\mathrm{x} .0=0$,for all $\mathrm{x} \in \mathrm{N}$ (from the left distributire law). Also, we will refer that all near rings and ideals in this work are left. 
Definition 2.5 [6]: Let I be an ideal of a near ring $N$, then I is called a completely prime ideal of $N$ if for allx, $y \in N$, $x . y \in I$ implies $x \in I$ or $y \in I$,denoted by c.p.I of $N$.

The $\mathrm{a} b-$ c.s. p. I near ring $\mathrm{N}$ in example (1.3) is not

Definition 2.6 [3]: Let $N$ be a near ring, I be an ideal of $N$ and letb $\in N$, then I is called a completely ideal with respect to the element $b$ denoted by $(b-c . p . I)$ of $N$, iffor all $x, y \in N, b .(x . y) \in I$ implies $x \in I$ or $y \in I$

Definition 2.7 [7]: A near ring $N$ is called an integral domain if $N$ has non_zero divisors.

Definition 2.8 [7]: Let $\left(N_{1},+,.\right)$ and $\left(N_{2}, \dot{+},:\right)$ be two near rings, the mapping $f: N_{1} \rightarrow N_{2}$ is called a near ring homomorphism if for all $m, n \in N_{1}$

$\mathrm{f}(\mathrm{m}+\mathrm{n})=\mathrm{f}(\mathrm{m}) \dot{+} \mathrm{f}(\mathrm{n})$ and $\mathrm{f}(\mathrm{m} . \mathrm{n})=\mathrm{f}(\mathrm{m}): \mathrm{f}(\mathrm{n})$

Definition 2.9 [7]: Anon-empty set $N$ is said to be a near field if $N$ is defined by two binary operations "+" and"." such that

1. $(\mathrm{N},+)$ Is a group

2. $(\mathrm{N} \backslash[0],$.$) Is a group$

3. $a \cdot(b+c)=a \cdot b+a \cdot c$, for all $a, b, c, \in N$.

Definition 2.10 [7]: The near ring $(N,+,$.$) is said to be a smarandache near ring denoted by (s-near ring) if it has$ aproper subset $M$ such that $(M,+,$.$) is a near field.$

Definition 2.11 [7]: Let $N$ be s-near ring. A normal subgroup I of $N$ is called a smarandache ideal (s-ideal) of $N$ related $M$ if,

i. $\quad$ For all $x, y \in M$ and for all $i \in I, x(y+i)-x y \in I$,

Where $\mathrm{M}$ is the near field contained inN.

ii. $\quad \mathrm{IM} \subseteq \mathrm{I}$

Remark 2.12 [7]: Let $\left[\mathrm{I}_{\mathrm{i}}\right]_{\mathrm{i} \in \mathrm{I}}$ be a chain of s-ideals related to a near field $\mathrm{M}$ of a near ring $\mathrm{N}$, then $\left[\mathrm{I}_{\mathrm{i}}\right]_{\mathrm{i} \in \mathrm{I}}$ Is a s-ideals related to near field $\mathrm{M}$

Remark 2.13 [6]: Let $\left(N_{1},+,.\right)$ and $\left(N_{2}, \dot{+},:\right)$ be two s-near rings and let $f: N_{1} \rightarrow N_{2}$ Be an epimomorphism and $N_{1}$ has $M_{1}$ as near filed. Then $M_{2}=f\left(M_{1}\right)$ is a near field of $N_{2}$.

Proposition 2.14 [4]: Let $\left(N_{1}, \grave{+},:\right)$ and $\left(N_{2}, \dot{+},:\right)$ be two s-near rings and $f: N_{1} \rightarrow N_{2}$ Be an epimomorphism and let $I$ be a

S-ideals related to a near field $\mathrm{M}$ of a near ring $\mathrm{N}$, and then $f(I)$ is s-ideals related to a near field $f(M)$.

Proposition 2.15 [4]: Let $\left(N_{1},+,.\right)$ be a s-near ring has a near filed $M_{1}, N_{2}$ be a s-near ring, $f: N_{1} \rightarrow N_{2}$ be an epimomorphism and let $J$ be s-ideals related to a near field $M_{2}$ of $N_{2}$, where $f\left(M_{1}\right)=M_{2}$ of $N_{2}$, then $f^{-1}(J)$ is a s-ideals related to a near field $M_{1}$ of $N_{1}$.

Definition 2.16 [7]: Let $N$ is an s-near ring. The s-ideals I related to a near field $M$ is called completely prime related to a near field $M$ of $N$ if, for all $x, y \in M, x . y \in I$ implies $x \in I$ or $y \in I$.denoted by (s.c.p.I) of $N$.

\section{The main results}

In this section, we define the notion of smarandache completely ideal with respect to an element $b$ (b-s.c.p.I) And study some properties of this notion, we will discuss the image and pre image of $b-s . c . p . I$ under near rings epimomorphism and explain the relationships between it and b-s.c.p.I of a near ring.

Definition 3.1: A s-ideals related to a near field $M$ of a s-near ring $N$ is called a samarandache completely ideal with respect to an element $b$ of $N(b-s . c . p . I)$, if b. (x.y) $\in$ I implies $x \in I$ or $y \in I$ for all $x, y \in M$.

Example 3.2: The left s-near ring with addition and multiplication defined by the following tables.

\begin{tabular}{ccccc}
\hline+ & 0 & $\mathrm{a}$ & $\mathrm{b}$ & $\mathrm{c}$ \\
\hline 0 & 0 & $\mathrm{a}$ & $\mathrm{b}$ & $\mathrm{c}$ \\
$\mathrm{a}$ & $\mathrm{a}$ & 0 & $\mathrm{c}$ & $\mathrm{b}$ \\
$\mathrm{b}$ & $\mathrm{b}$ & $\mathrm{c}$ & 0 & $\mathrm{a}$ \\
$\mathrm{c}$ & $\mathrm{c}$ & $\mathrm{b}$ & $\mathrm{a}$ & 0 \\
\hline
\end{tabular}




\begin{tabular}{ccccc}
\hline$\cdot$ & 0 & $\mathrm{a}$ & $\mathrm{b}$ & $\mathrm{c}$ \\
\hline 0 & 0 & 0 & 0 & 0 \\
$\mathrm{a}$ & 0 & $\mathrm{a}$ & $\mathrm{a}$ & 0 \\
$\mathrm{~b}$ & 0 & $\mathrm{~b}$ & $\mathrm{c}$ & $\mathrm{c}$ \\
$\mathrm{c}$ & 0 & 0 & $\mathrm{c}$ & $\mathrm{c}$ \\
\hline
\end{tabular}

The s-ideal $I=[0, a]$ related to the near field $M=[0, c]$ is $b-$ s.c.p. $I$ of $N$ since $0 .(c . c)=0 \in I, b u t c \notin I$.

Proposition 3.3: Let I be a s-ideal related to a near field $\mathrm{M}$ of a s-near ring $\mathrm{N}$,then I is a s. c. p. I of $\mathrm{N}$ if and only if I is 1 - s. c. p. I, where 1 is the multiplicative identity element of $\mathrm{M}$.

Proof: Suppose I is a s. c.p. I ideal of N

And let $\mathrm{x}, \mathrm{y} \in \mathrm{M}$ such that1. (x.y) $\in \mathrm{I}$.

Then we have 1. (x.y) = x.y $\in$ I

$\Rightarrow \mathrm{x} \in \mathrm{I}$ or $\mathrm{y} \in \mathrm{I}$ [Since $\mathrm{I}$ is a s. c. p. I ofN].

$\Rightarrow \mathrm{I}$ is $1-$ s.c.p. I Of $\mathrm{N}$.

Conversely,

Let $\mathrm{x}, \mathrm{y} \in \mathrm{M}$ such that $\mathrm{x} . \mathrm{y} \in \mathrm{I}$

$\Rightarrow \mathrm{x} . \mathrm{y}=1$. (x. $\mathrm{y}) \in \mathrm{I} \Rightarrow \mathrm{x} \in \mathrm{I}$ or $\mathrm{y} \in \mathrm{I}$ [Since $\mathrm{I}$ is 1. (x. $\mathrm{y})$ of $\mathrm{N}$ ].

Remark 3.4: In general an S.C.P.I related to a near field $\mathrm{M}$ of an s-near ring $\mathrm{N}$ may not be b-S.C.P.I related to $\mathrm{M}$ of $\mathrm{N}$ as in the following example

Example 3.5: Consider the s-near ring of integers mod $6(\mathrm{z} 6, \mathrm{t} 6, .6)$; the s-ideal $\mathrm{I}=[0,2,4]$ is S.C.P.I related to the near field $\mathrm{M}=[0,3]$, but it is not 2-S.C.P.I of $\mathrm{N}$, since $3 \in \mathrm{M}$ and 2.(3.3)=0 $\in \mathrm{I}$ but $3 \notin \mathrm{T}$.

Proposition 3.6: Let I be a b-C.P.I related to a near field $\mathrm{M}$ of a s-near ring N. then I is a b-S.C.P.I of N.

Proof: Let $x, y \leftarrow M$, such that b. (x.y) $\in$ I

$\Rightarrow \mathrm{x}, \mathrm{y} \in \mathrm{N}[$ since $\mathrm{M}$ is a proper subset of $\mathrm{N}]$

$\Rightarrow \mathrm{x} \in \mathrm{I}$ or $\mathrm{y} \in \mathrm{I}$ [since $\mathrm{I}$ is b-S.C.P.I of $\mathrm{N}$ ]

$\Rightarrow \mathrm{I}$ is a b-S.C.P.I of N.

Remark (3.7): The conzerse of proposition (3.6) may not be true as in the following example.

Example 3.8: Consider the s-near ring of integers mod 12 (Z12, t12, i12); s-ideal $\mathrm{I}=[0,2,4,6,8,10]$ if z-S.C.P.I related

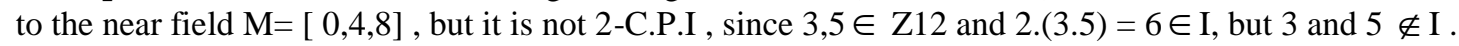

Proposition 3.9: Let $\mathrm{N}$ be a s-near ring and let $\mathrm{I}$ be a s-ideal related to a near field $\mathrm{M}$ of $\mathrm{N}$. then $\mathrm{I}$ is a b-S.C.P.I of $\mathrm{N}$ if and only if $\mathrm{M}$ is a subset of $\mathrm{I}$, for all $\mathrm{b} \in \mathrm{I}>$

Proof: Suppose I is a b-S.C.P.I, $b \in I$ and $X \in M$.

Now,

$\mathrm{X}^{2}=\mathrm{x} . \mathrm{x} \in \mathrm{I}, 0 \in \mathrm{I}$ and $0 . \mathrm{x}^{2}=0 .(\mathrm{x} . \mathrm{x})=0 \in \mathrm{I}$

$\mathrm{x} \in \mathrm{I}$ [since $\mathrm{I}$ is o-S.C.P.I ],

$\Rightarrow \mathrm{M}$ is a subset of I

Conversely,

Let $\mathrm{b} \in \mathrm{I}$ and $\mathrm{x}, \mathrm{y} \in \mathrm{M}$ such that b.(x.y) $\in \mathrm{I}$

$\Rightarrow \mathrm{x}$ or $\mathrm{y} \in \mathrm{I}$ [since $\mathrm{M} \subseteq \mathrm{I}$ ]

$\Rightarrow \mathrm{I}$ is b-S.C.P.I of N.

Proposition 3.10: Let $\mathrm{N}$ be a s-near integral domain . then $\mathrm{I}=[0]$ is b-S.C.P.I related to a near field $\mathrm{M}$ of $\mathrm{N}$, for all $\mathrm{n} \in$ Nl [0] .

Proof: Let $b \in \mathrm{Nl}[0]$ and $\mathrm{x}, \mathrm{y} \in \mathrm{M}$, such that b.(x.y) $\in \mathrm{I}$

$\Rightarrow$ b. $(x . y)=0$

$\Rightarrow \mathrm{x} . \mathrm{y}=0$ [since $\mathrm{b} \neq 0$ and $\mathrm{N}$ is a near integral domain]

$\Rightarrow \mathrm{x}=0$ or $\mathrm{y}=0 \Rightarrow \mathrm{x} \in \mathrm{I}$ or $\mathrm{y} \in \mathrm{I}$

$\Rightarrow \mathrm{x} \in \mathrm{I}$ or $\mathrm{y} \in \mathrm{I}$.

$\Rightarrow \mathrm{I}$ is a b-S.C.P.I of N. 
Proposition 3.11: Let $\mathrm{N}$ be a zero symmetric s-near ring and let $\mathrm{I}=[0]$. Then $\mathrm{I}$ is not o-S.C.P.I of $\mathrm{N}$ related to all near fields of $\mathrm{N}$.

Proof: Suppose I is o-S.C.P.I related to a near field $\mathrm{M}$ of $\mathrm{N}$.

Since $\mathrm{M}$ is a near field $\Rightarrow \mathrm{M} \neq[0]$

$\Rightarrow \exists X \in M$, such that $X \neq 0$.

Now,

$0 \mathrm{x}^{2}=0 .(\mathrm{x} . \mathrm{x})=0 \in \mathrm{I}$

$\Rightarrow \mathrm{x} \in \mathrm{I} \Rightarrow \mathrm{x}=0$ and this contradiction [ since $\mathrm{x} \neq 0$ ]

$\Rightarrow \mathrm{I}$ is not 0- S.C.P.I related to $\mathrm{M}$ of $\mathrm{N}$.

Proposition 2.12: Let $\mathrm{N}$ be a s-near ring and let [Ii] $]_{i} \in_{\mathrm{I}}$ be a chain of b-S.C.P.I related to a near field $\mathrm{M}$ of $\mathrm{N}$, for all $\mathrm{i}$ $\in I$. then $V_{i} \in I_{i}$ is a b-S.C.P.I related to $M$ of $N$.

Proof : Since $[\mathrm{Ii}]_{\mathrm{i}} \in_{\mathrm{I}}$ is a chain a b-S.C.P.I related to $\mathrm{M}$ of $\mathrm{N}$.

$\Rightarrow \mathrm{Ii}$ is a s-ideal of $\mathrm{N}$ for all $\mathrm{i} \in \mathrm{I}$.

$\Rightarrow \mathrm{V}_{\mathrm{i} \in \mathrm{I}} \mathrm{Ii}$ is a s-deal of $\mathrm{N}$ [ By remark (2.12)]

Now,

Let $x, y \in M$, such that b.(x.y) $\in V_{i} \in{ }_{I} I_{i}$

$\Rightarrow$ There exists b-S.C.P.I related $\mathrm{M} \mathrm{I}_{\mathrm{k}} \in[\mathrm{Ii}]_{\mathrm{i}} \in{ }_{\mathrm{I}}$ of $\mathrm{N}$, such that b.(x.y) $\in \mathrm{I}_{\mathrm{k}}$

$\Rightarrow \mathrm{x} \in \mathrm{I}_{\mathrm{k}}$ or $\mathrm{y} \in \mathrm{I}_{\mathrm{k}}$ [ since $\mathrm{I}_{\mathrm{k}}$ is a b-S.C.P.I of $\mathrm{N}$ ]

$\Rightarrow \mathrm{x} \in \mathrm{V}_{\mathrm{i}} \in{ }_{\mathrm{I}} \mathrm{I}_{\mathrm{i}}$ or $\mathrm{Y} \in \mathrm{V}_{\mathrm{i}} \in \mathrm{I}_{\mathrm{I}} \mathrm{I}_{\mathrm{i}} \Rightarrow \mathrm{V}_{\mathrm{i}} \in{ }_{\mathrm{I}} \mathrm{I}_{\mathrm{i}}$ is a b-S.C.P.I of $\mathrm{N}$.

Remark 3.13: In general, if [Ii] ${ }_{i} \in_{I}$ is a family of b-S.C.P.I related to a near field $M$ of as near ring $N$, then $\bigcap_{i \in I} I_{i}$ and $\mathrm{V}_{\mathrm{i}} \in{ }_{\mathrm{I}} \mathrm{I}_{\mathrm{i}}$ may not be b-S.C.P.I

Related to $\mathrm{M}$ of $\mathrm{N}$, as in the following example

Example 3.13: Consider the s-near ring of integer's mod12. $(\mathrm{Z} 12, \mathrm{t} 12,12)$, the s-ideals $\mathrm{I}=[0,6]$ and $\mathrm{J}=[0,4,8]$ are 3 S.C.P.I. related to the near field $\mathrm{M}=[0,4,8]$ of $\mathrm{Z} 12$, but the s-ideal $\mathrm{I} \cap \mathrm{J}=[0]$ is not 3-S.C.P.I related to $\mathrm{M}$ of $\mathrm{Z} 12$, since 3.(3.8)=0 $\in \mathrm{I}$, but and $8 \notin \mathrm{I}$, Also , the subset $\mathrm{I} \cup \mathrm{J}=[0,4,6,8]$ is s-ideal of $\mathrm{Z} 12$ and this implies $\mathrm{I} \cup \mathrm{J}$ is not 3-S.C.P.I related to $\mathrm{M}$ of $\mathrm{Z} 12$.

Theorem 3.15: Let $(N 1, *, 0)$ and $(N 2, t, 0)$ be two s-near rings, $F: N 1 \rightarrow N 2$ be an epimvor phism and let I be a $b$ S.C.P.I related to near field $M$ of $N$, then $f(I)$ is $f(b)-S . C . P . I$ related to the near field $f(M)$ of $N 2$.

Proof :By remark (2.13), we have $\mathrm{f}(\mathrm{I})$ is a s-ideal related to a near field $\mathrm{f}(\mathrm{M})$

Now Let $\mathrm{f}(\mathrm{m} 1), \mathrm{f}(\mathrm{m} 2) \in \mathrm{f}(\mathrm{m})$, such that

$f(b) !(f(m 1) ! f(m 2) \in f(I)$

$\Rightarrow \mathrm{f}(\mathrm{b}(\mathrm{m} 1 . \mathrm{m} 2)) \in \mathrm{f}(\mathrm{I})$

$\Rightarrow \mathrm{f}(\mathrm{b}(\mathrm{m} 1 . \mathrm{m} 2)) \in \mathrm{f}(\mathrm{I})$

$\Rightarrow$ either $\mathrm{m} 1 \in \mathrm{I}$ or $\mathrm{m} 2 \in \mathrm{I}$ or $\mathrm{m} 2$ [ since $\mathrm{I}$ is b- S.C .P. I related to $\mathrm{M}$ of N1 ]

$\Rightarrow \mathrm{f}(\mathrm{m} 1) \in \mathrm{f}(\mathrm{I})$ or $(\mathrm{m} 2) \in \mathrm{f}(\mathrm{I})$

$\Rightarrow \mathrm{f}(\mathrm{I})$ is a $\mathrm{f}(\mathrm{b})$ - S.C.P. I related to $\mathrm{f}(\mathrm{M})$ of $\mathrm{N} 2$

Theorem 3.16: Let $(N 1,+$, . ) be as - near ring has a near field $M 1$, ( N2) be $S$ - near ring, $f: N 1 \rightarrow N 2$ be an epimomorphism, and Let $J$ be a $b$-S.C.P.I related to the near field $f(M)$ of $N 2$, then $f^{l}$ (I) is a - S.C.P.I related to a near field $M$ of $N 1$, where $b-f(a)$.

Prof: By proposition ( 2.15), we have $\mathrm{f}^{-1}(\mathrm{~J})$ is a $\mathrm{S}$ - ideal related to $\mathrm{M}$ of $\mathrm{N} 1$. Now, Let $\mathrm{x}, \mathrm{y} \in \mathrm{M}$, such that a. (x.y ) $\in$ $\mathrm{f}-1(\mathrm{~J})$

$\Rightarrow \mathrm{f}(\mathrm{x}), \mathrm{f}(\mathrm{y}) \in \mathrm{f}(\mathrm{M})$ and $\mathrm{f}(\mathrm{a} !(\mathrm{x} \mathrm{y}) \in \mathrm{J}$

$\Rightarrow \mathrm{f}(\mathrm{x}), \mathrm{f}(\mathrm{y}) \in \mathrm{f}(\mathrm{M})$ and $\mathrm{f}(\mathrm{a}) ! \mathrm{f}(\mathrm{x}), \mathrm{f}(\mathrm{y})) \in \mathrm{J}$

$\Rightarrow$ either $\mathrm{f}(\mathrm{x}) \in \mathrm{J}$ or $\mathrm{f}(\mathrm{y}) \in \mathrm{J}[$ since $\mathrm{J}$ is b- S.C.P. I related to $\mathrm{f}(\mathrm{M})$ of N2 ]

$\Rightarrow$ either $\mathrm{x} \in \mathrm{f}^{-1}(\mathrm{~J})$ or $\mathrm{y} \mathrm{f}^{-1}(\mathrm{~J})$ or $\mathrm{y} \in \mathrm{f}^{-1}(\mathrm{~J})$

$\Rightarrow \mathrm{f}^{-1}(\mathrm{~J})$ is a b- S.C.P. I related to $\mathrm{f}(\mathrm{M})$ of $\mathrm{N} 2$

Corollary 3.17: Let $(\mathrm{N} 1,+, 0)$ be a $S-$ near ring has a near field M, ( N2 , +' , ." ) be a S- near ring, f : N1, $\rightarrow$ N2 be an e pimomorphism, and if $\left[\mathrm{o}^{1}\right]$ be a b- S.C .P. I related to the near field $\mathrm{f}(\mathrm{M})$ of N2 The ker(f) is b- S.C .P. I related to a near field $\mathrm{M}$ of $\mathrm{N} 1$, where 
$\operatorname{Ker} f=[x \in N 1: f(x)=0]$ and $b=f(a)$

Proof: Since $\mathrm{f}^{-1}\left(\left[0^{1}\right)\right] \mathrm{l}=\mathrm{ker}(\mathrm{f})$, then where Rer (f) is a - S.C .P. I related to M of N1

[By theorem (3-16)]

\section{References}

[1] G. Pilz, "Near Ring ", North Holland Publ and Co., 1977.

[2] H.A. Abujabal, M.A obaid and M.A. han, "On structure and Commutatitiy of near Rings", An to fagasta - chule, 2000.

[3] H.H Abbass and M.A. Who Mmed, "On alompletely prime Ideal with respect to an element of a near ring", J. of Kerbala university. Vol. 10, No 3, 2012.

[4] H.H Abbass and S.M. Ibrahem, "On Fuzzy completely semi orime Ideal with respect to an element of a near Ring", M Sc. Thesis university of Kufa 2011.

[5] N.J Groenewatd, "The completely prim radical near rings", Acta Math. Hung, VO 133, 1988.

[6] P. Dheena and G. Sathesh Kumar, "Completely z-prime Ideal in near ring", India, 2007.

[7] W.B Vasantha and A. Samy, "Samarandache near ring", U.S. America research press, 2002. 